This is a postprint version of the following published document:

Ripoll, J.; Koberstein-Schwarz, B.; Ntziachristos, V. Unleashing optics and optoacoustics for developmental biology. Trends in biotechnology, 33(11), pp. 679-691, November 2015

DOI: https://doi.org/10.1016/j.tibtech.2015.08.002

(C) 2015 Published by Elsevier Ltd.

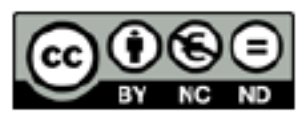

This work is licensed under a Creative Commons Attribution-NonCommercialNoDerivatives 4.0 International License. 


\section{Unleashing Optics and Optoacoustics for Developmental Biology}

J. Ripoll, ${ }^{1,2}$ B. Koberstein-Schwarz, ${ }^{3,4}$ and V. Ntziachristos ${ }^{4, *}$

The past decade marked an optical revolution in biology: an unprecedented number of optical techniques were developed and adopted for biological exploration, demonstrating increasing interest in optical imaging and in vivo interrogations. Optical methods have become faster and have reached nanoscale resolution, and are now complemented by optoacoustic (photoacoustic) methods capable of imaging whole specimens in vivo. Never before were so many optical imaging barriers broken in such a short time-frame: with new approaches to optical microscopy and mesoscopy came an increased ability to image biology at unprecedented speed, resolution, and depth. This review covers the most relevant techniques for imaging in developmental biology, and offers an outlook on the next steps for these technologies and their applications.

The Challenges of In Vivo Imaging

One of the most evident needs in ontogeny is the need to recover volumetric information at cellular resolution of a live organism as it develops. This need has been one of the main driving forces of most of the novel imaging approaches. While probing live tissue, our main interest lies in maintaining the conditions as physiologically normal as possible. In this regard, maintained exposure to light not only causes photobleaching of the fluorescent proteins expressed by the organism but also has adverse phototoxic effects which thwart normal development. The amount of research and technology devoted to reducing this exposure is evident in the evolution of confocal microscopy where the use of the spinning disk [1] in the past decade provided the means to extend the number of hours the samples could be under the microscope.

Photobleaching, of course, is not the only problem we encounter when imaging a developing organism. As the cellular structure of our specimen changes, so do its optical properties, the most relevant of which is scattering (Box 1), which scrambles the spatial information yielding a 'blurry' image (and conversely, a widening of the excitation focal spot within the sample). This occurs mainly when samples have reached a size comparable to the transport mean free path (Box 1) which, in the context of in vivo imaging, refers to sample sizes not in the micron range, but rather in the tenths or hundreds of microns and above.

In summary, our ability to image a developing organism from the first cell division to the adult stages depends on how we address three main aspects of imaging: penetration depth (and therefore the effect of scattering); speed (resulting in lower photobleaching which in turn allows longer time-lapse measurements, and the possibility of imaging in 3D fast processes such as the fate of individual cells); and resolution (our ability to spatially resolve individual specific markers). The approaches taken to address these three parameters have resulted in a large number of

\section{Trends}

The use of the NIR II (1000 $1700 \mathrm{~nm})$ range of the spectrum will open new ways to image deeper in vivo with unprecedented spatial resolution.

Multispectral optoacoustic tomogra phy is capable of imaging specific fluor ophores and chromophores through several centimeters of tissue while maintaining resolution under $100 \mu \mathrm{m}$.

Novel light sheet microscopy approaches are capable of 3D imaging in small transparent specimens with subcellular resolution at rates of 200 sections per second.

New advances in super resolution approaches can image live cells with resolution under $100 \mathrm{~nm}$ and offer 3D imaging capabilities.

${ }^{1}$ Department of Bioengineering and Aerospace Engineering, Universidad Carlos III de Madrid, 28911 Madrid, Spain

${ }^{2}$ Experimental Medicine and Surgery Unit, Instituto de Investigación Sanitaria Gregorio Marañón, 28007 Madrid, Spain

${ }^{3}$ Carl Zeiss AG, Corporate Research and Technology, Carl Zeiss Promenade 10, 07745 Jena, Germany ${ }^{4}$ Institute of Biological and Medical Imaging (IBMI), Technische Universität München and Helmholtz Zentrum München, Ingolstädter Landstrasse 1, 85764 Neuherberg, Germany

${ }^{*}$ Correspondence:

v.ntziachristos@helmholtz muenchen. de (V. Ntziachristos). 
Box 1. Scattering and Its Effect on In Vivo Imaging

As a small cluster of cells, which exhibits negligible scattering, grows and expands, so does their scattering power. This has, and still does, limit the age and/or size we are able to study with cellular resolution in vivo. Once the scattering contribution of the developing organism is significant, either through growth or the creation of more complex interfaces, our excitation sources are not capable of focusing within the organism, and our optical detection methods are not capable of imaging the fluorescence emitted with the required spatial resolution.

Once we reach a specific tissue density, and the contribution of scattering is substantial, most of the approaches based on geometrical optics fail, even when combined with multiphoton excitation and/or structured illumination. This tissue density, or rather, the optical distance light has to travel to have a strong scattering component, is approximately $\sim 1 \mathrm{~mm}$ in tissues, this distance being termed the 'transport mean free path'. In these cases we need to resort to mesoscopic methods, which can account for the effect of scattering, or to other methods such as optoacoustic tomography for which the effect of scattering is several orders of magnitude smaller.

It is important to understand that the 'optical size' of a specimen is measured in terms of its size in transport mean free path units: samples with dimensions much smaller than the transport mean free path are considered to be transparent, while samples larger than the transport mean free path are considered to cause diffusion of light. Techniques such as wavefront correction attempt to correct the distortion of the initial coherent illumination wavefront introduced by scattering. In light microscopy, spatial light modulators (SLM) [86 90] and adaptive optics are capable of modulating the phase and intensity, and therefore the wavefront of the incident beam. These wavefront correcting techniques have several potential applications where, for example, the corrected focus allows them to effectively remove the effect of scattering over large volumes $(>240 \mu \mathrm{m})$ of the live zebrafish brain [91].

technological developments and data-processing schemes that offer diverse potential for imaging a developing organism. Nonetheless, navigating the imaging features and application possibilities of these methods can be a challenging task. With the fundamental assumption that there is no single imaging approach that will address all issues simultaneously, in this review we offer a classification of recent developments in imaging techniques, in the hope of aiding the selection of 'the right tool for the job'. This classification is based on which aspect of imaging (depth, speed, or resolution) is tackled more efficiently, discussing the pros and cons of each technique when applied specifically for imaging in developmental biology.

\section{Imaging Deeper}

The optical properties of the tissue under investigation, mainly absorption and scattering, will determine how light will propagate and in turn establish our resolution and sensitivity [2]. The attenuation of light in tissue is mainly caused by the absorption of light by hemoglobin and water, blood being highly absorbing in the visible wavelengths (up to $\sim 650 \mathrm{~nm}$ ), while water has a high increase in absorption which starts in the infrared (IR) portion of the spectrum (from $1100 \mathrm{~nm}$ onwards). The spectral range in the near-infrared (NIR), where the absorption of both hemoglobin and water are minimum, is termed an 'optical window', allowing increased imaging depth note that there is also a narrow second NIR window at 1300-1400 nm, termed the NIR lla window [3]. However, even though scattering exhibits lower values as we go to longer wavelengths, unfortunately there is no such thing as a completely 'scattering-free window'. In this aspect, Hong et al. have made significant developments in using the intrinsic photoluminescence of single-walled nanotubes in the NIR lla spectral window to image through the intact skull of a mouse at the unprecedented resolution of sub- $10 \mu \mathrm{m}$ at $2 \mathrm{~mm}$ depth. This second optical window has yet to be exploited and shows great potential because it significantly lowers the contribution of scattering. With respect to the traditional optical window, to achieve penetration depths in the order of the transport mean free path ( $1 \mathrm{~mm}$ in tissues, Box 1) or higher, we can therefore either exploit the lower scattering and absorption present in tissue by using $\mathbb{R}$ wavelengths, as in multi-photon microscopy, or we may physically model light propagation in tissues and account for the presence of scattering as in projection tomography. For larger penetration depths which enable whole-body in vivo imaging, the effect of scattering is circumvented by combining the advantages of optics and acoustics as in optoacoustic imaging, which consist of the measurement of an acoustic signal generated by the absorption of light. 


\section{Multiphoton Microscopy}

Multiphoton microscopy generates contrast by making use of nonlinear optical processes which take place when high energy densities are achieved [4,5], a situation which requires the use of short pulsed sources (in the femtosecond range - the shorter the pulse, the higher the energy density). Of the different non-linear effects, the most widely used is two-photon excitation microscopy (2PM), where the fluorescence in the sample is excited through the simultaneous absorption of two photons of lower energy, an event which requires high energy flux. This high energy density is maximum at the focal point, providing optical sectioning and reducing the background signal. By scanning the focal spot through the volume of the sample, in what is termed 'laser scanning microscopy', an image of the sample is generated. In addition to optical sectioning and an inherently higher resolution owing to non-linear excitation, the use of wavelengths in the IR ensures penetration depths of up to $1 \mathrm{~mm}$, an additional advantage of 2PM. With this technique structures deep inside biological tissue can be resolved with $\sim 1.9 \mu \mathrm{m}$ resolution, as seen in Figure $1 \mathrm{~A}$ where an image of cortical vasculature in a mouse brain is shown [6].

Three-photon excitation microscopy (3PM), which makes use of excitation wavelengths in the 1600-1800 nm region (a region between two high absorption peaks of water of the NIR II part of the spectrum), and in which three photons are absorbed simultaneously, may be used to improve spatial resolution and a slight increase in depth penetration. Horton et al. [7] demonstrated in mice that with 3PM it is possible to resolve neuronal structures in regions of the brain deep under the surface, with $\sim 0.9 \mu \mathrm{m}$ and $\sim 4.4 \mu \mathrm{m}$ lateral and axial resolution, respectively.

In addition to depth penetration, simultaneous imaging of multiple fluorescent proteins is also a crucial need in live imaging. In [8] an optical parametric oscillator is used to generate a beam with a tunable wavelength above $1080 \mathrm{~nm}$, allowing excitation in the NIR. Simultaneous two-photon excitation of three fluorophores has also been achieved [9] by combining two beams of different wavelengths, generating the third excitation wavelength through the spatiotemporal overlap between both beams. The simultaneous excitation of three distinct fluorophores enabled imaging of Brainbow-labeled mouse cortical tissue [10], allowing the differentiation of individual neurons within the tissue.

Exploitation of additional non-linear effects such as second or third harmonic generation (SHG) THG) can also be used to generate label-free tissue contrast, but requires the presence of specific structures in the tissue that are capable of generating harmonic frequencies in response to the excitation frequency. Elongated and periodic structures such as myofibrils or motitic spindles, for example, are capable of SHG, generating light of exactly half the wavelength (or one third of the original wavelength for THG) [11]. The structural contrast obtained by SHGM and THGM can provide complementary information to the images obtained in 2PM, which is why the two methods are often combined [7,9,12]. Although both SHG and THG microscopy have been frequently used since the turn of the millennium, only recent developments have applied them to cell lineage tracking in early zebrafish embryos [13] or to live brain imaging combined with targeted patch-clamp recordings of single neurons, allowing both recording and influencing the membrane potential of single cells [12] while performing volumetric imaging.

\section{Projection Tomography}

Optical volumetric images can be also generated by collecting light transmitted through the volume of interest at different projections (angles), in analogy to X-ray computed tomography (CT). The technique was originally termed optical projection tomography [14-16], and was first published as a technique capable of imaging cleared and fixed tissues, but was later also applied to microscopic samples in vivo [17-20]. Image formation is based on using mathematical models to reconstruct optical contrast within the medium being imaged, an approach capable of 


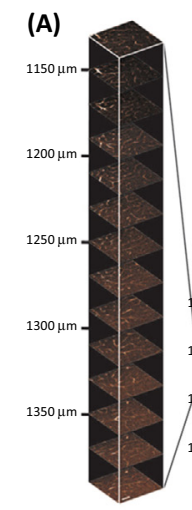

(E)

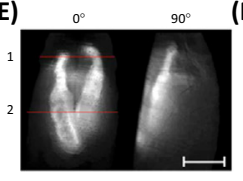

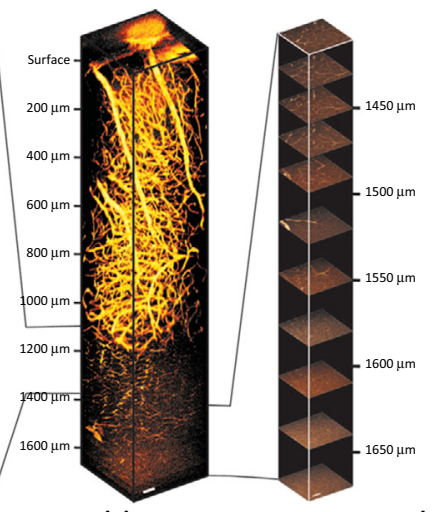

(F)

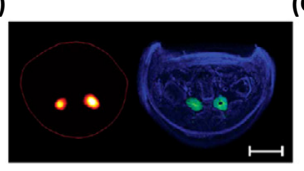

(B)

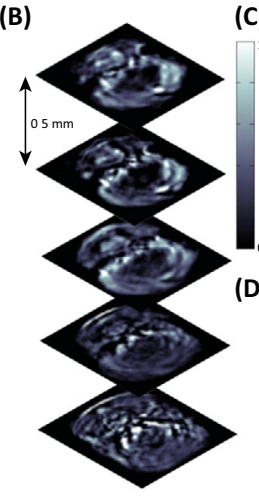

(G)

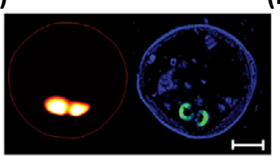

(C)

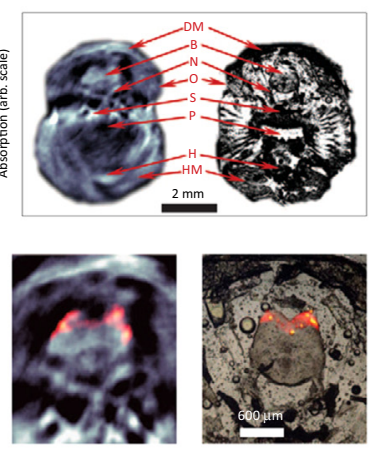

(H)

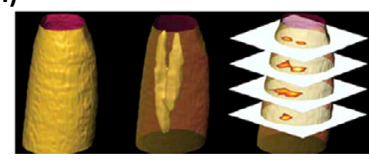

Trends in Biotechnology

Figure 1. (A) Two photon fluorescence images of cortical vasculature in a mouse brain. $235 x$ y frames from $60 \mu \mathrm{m}$ above the cortical surface to $1110 \mu \mathrm{m}$ below are taken at a depth increment of $5 \mu \mathrm{m}$. The depth increments in the stack are $20 \mu \mathrm{m}$ from 1110 to $1490 \mu \mathrm{m}$ and $30 \mu \mathrm{m}$ from 1490 to $1670 \mu \mathrm{m}$. These increments are adjusted to keep the total imaging time at a practical limit while covering all the available depths. 3D reconstruction is made in ImageJ software using the volume viewer plug in. Expanded 3D stacks are shown for the deepest sections (>1130 $\mu \mathrm{m}$ ). Scale bar $50 \mu \mathrm{m}$ (from Kobat et al. [6]) (B D) $3 \mathrm{D}$ in vivo imaging through the brain of an adult (6 month old) mCherry expressing transgenic zebrafish. (B) Five transverse opto acoustic imaging slices through the hindbrain area at the level of crista cerebellaris of a living zebrafish taken at $585 \mathrm{~nm}$. (C) Example of an imaged slice (left) and its corresponding histological section in inverted colors (right). Abbreviations: B, hindbrain; DM, dorsal fin musculature; $\mathrm{H}$, heart; $\mathrm{HM}$, hypobranchial musculature. $\mathrm{N}$, lateral line nerve; O, operculum; $\mathrm{P}$, pharynx; S, skull bones. (D) MSOT image of the brain (enlarged) with mCherry expression shown in color and the corresponding fluorescent histology of a dissected fish at the hindbrain level (from Razansky et al. [34]). (E H) In vivo reconstructions of $D$. melanogaster salivary glands (with mesoscopic fluorescence tomography). (E) In vivo reconstructions of the pupal case and the GFP expressing salivary glands of a D. melanogaster prepupa (projections at $0^{\circ}$ and $90^{\circ}$ ). Scale bar, $500 \mu \mathrm{m}$. Red lines indicate the planes where the reconstructions were obtained. $(F, G)$ Planar reconstructions of the salivary glands and corresponding histology analysis (blue, DAPI staining; green, GFP fluorescence) obtained in planes 1 and 2, respectively. The histology images were acquired from morphologically matched areas of different pupae at the same developmental stage. Scale bars, $300 \mu \mathrm{m}$. (H) Rendering of the pupal case and the salivary glands. White planes show different planar reconstructions obtained at different heights (from Vinegoni et al. [23]).

imaging fluorescence and absorption. When imaging elongated samples the specimens may be stacked and translated vertically while imaging following a helical motion. This is the approach used in helical optical projection tomography [21] (hOPT), an approach that has also been recently used for long time-lapse imaging of the head eversion in Drosophila development, and for high-throughput imaging [22].

Both OPT and hOPT, however, rely on the filtered back-projection algorithm traditionally used in CT to generate a 3D image, and therefore suffer a degradation of their performance proportion to the amount of scattering the specimen presents. To account for this, mesoscopic fluorescence tomography (MFT) [23] was suggested, using more sophisticated light propagation models to account for the presence of scattering in the measurements, improving imaging performance in scattering samples compared to OPT [23], for example in in vivo imaging of Drosophila melanogaster development (Figure 1E-H). Another approach to improve resolution while imaging in the presence of scattering is time-gated OPT [18,24], which measures early arriving photons to remove the contribution of scattering. Fieramonti et al. also introduced an OPT system capable of imaging in 3D the distribution of flow in zebrafish [25]. 
The main advantage of OPT, hOPT, and MFT is the fact that, if scattering is present, there is a way to account for it in the inverse model because all spatial information (despite being scrambled due to scattering) is still present in the measurements. Another great advantage of projection methods is the fact that absorption may be used as a source of contrast, something which is not possible with confocal or laser sheet microscopy (see below). The main drawback of optical projection approaches, however, is that they typically require that a large part of the sample remains in focus during the measurement. This means that low numerical apertures need to be used, in most cases by using an iris at the back focal plane of the objective, limiting the resolution the technique can achieve and also significantly lowering the sensitivity of the imaging setup, requiring long exposure times and high power, thus significantly exposing the specimen to phototoxic effects.

\section{Optoacoustic Imaging}

Opto- or photoacoustic imaging $(\mathrm{OA})$ is based on the generation of ultrasound waves within tissue due to the thermoelastic expansion of localized tissue after absorption of a pulse of light. By tracing the origin of the sound waves, tomographic methods [26,27] provide 3D images of optical absorption. Compared to light scattering of visible or NIR light, ultrasound is scattered several orders of magnitude less in tissue, resulting in depth penetration of several $\mathrm{cm}$, while maintaining a resolution of $\sim 100 \mu \mathrm{m}$ or less. Because hemoglobin presents strong light absorption in the visible, optoacoustic imaging is well suited for resolving vascular structures [28]. In addition, multispectral illumination at several wavelengths combined with spectral unmixing can reveal specific chromophores and fluorescent proteins, distinguishing between oxy- and deoxyhemoglobin, a technique termed multispectral optoacoustic tomography (MSOT) [29,30]. The large penetration depths and the relatively high resolution of $\sim 100 \mu \mathrm{m}$ (note that this resolution is one tenth of the transport mean free path, $\sim 1 \mathrm{~mm}$ ) make this approach ideal for macroscopic imaging of small animals [31-33]. Higher resolution might be achieved with optoacoustic tomography, as seen in Figure 1B-D where Razansky et al. [34] show how MSOT can be used to resolve fluorophores in the brain of an adult transgenic zebrafish in vivo with a scalable spatial resolution of a few tenths of microns. In addition, Gateau et al. have shown that 3D optoacoustic tomography enables imaging depths of several millimeters to centimeters with scalable resolution under $\sim 100 \mu \mathrm{m}$ [35]. Even higher resolution can be obtained, as shown by Omar et al., by making use of an ultra-wideband single element transducer with a bandwidth of $20-180 \mathrm{MHz}$ [36], demonstrating an axial resolution of $\sim 4 \mu \mathrm{m}$ and a lateral resolution of $\sim 18 \mu \mathrm{m}$ for imaging depths up to $5 \mathrm{~mm}$, allowing imaging of vascular structures under the skin or in small-animal model systems.

\section{Need for Speed: Imaging Faster}

Apart from depth, another main requirement for developmental studies is imaging speed, an issue that has been addressed directly by light sheet microscopy techniques [37], offering unprecedented full specimen imaging speeds suitable for in vivo imaging of fast processes such as the beating zebrafish heart [38-40]. These new fast volumetric imaging approaches now extend both the number of time-points and the time-window we can use for time-lapse imaging, reducing the risk of photobleaching and phototoxic effects in the specimen.

\section{Light Sheet Microscopy}

Light sheet microscopy or ultramicroscopy, originally developed in 1903 [41], reappeared in 1983 when it was applied to fixed and cleared tissue as orthogonal-plane fluorescence optical sectioning (OPFOS) [42], and as thin laser sheet imaging microscopy (TSLIM) in 2002 [43] finally being applied to whole fixed and cleared organs as ultramicroscopy [44]. However, it was not until Huisken et al. in 2004 [45] presented selective plane illumination microscopy (SPIM) data applied to in vivo zebrafish imaging that light sheet microscopy really took off. Since then there 
has been an explosion of papers devoted to light sheet microscopy, improving both the speed of acquisition and the resolution.

Light sheet microscopy is based on generating a sheet of light within the specimen and ensuring this sheet of light coincides with the focal plane of a high numerical objective (see [46] and the special issue of Nature Collections [37] for a review on light sheet microscopy). Its contrast is based on fluorescence and, for this geometry to take place we need to be able to access the sample from $90^{\circ}$. How one generates this light sheet will determine how sensitive the setup is to scattering. The original setups made use of cylindrical lenses to generate the light sheet, while further developments employed structured illumination such as digitally scanned light sheet microscopy (DSLM) [47] and DSLM-SI [48], sometimes combined with multi-photon excitation to improve resolution even further [49-51] (Figure 2A shows high-speed volumetric imaging of chromosomes in mitosis with sub-micron resolution).

There were two main drawbacks from the early light sheet microscopy setups: anisotropic resolution and a low optical sectioning resolution owing to the thickness of the light sheet. The former has been addressed by combining several views in multiview SPIM (mSPIM) [52,53], MuViSPIM [54], or dual-inverted SPIM (diSPIM) [55]. By contrast, low optical sectioning resolution has been recently addressed by altering the shape of the beam, noting that when using traditional Gaussian beams this focused light sheets imply smaller fields of view. To overcome this fact other beams have been explored, in particular Bessel beams [55] owing to their longer depth of focus and their 'self-reconstructing' capabilities which suffer less from the effect of scattering $[49,50]$. Recently, Vettenburg et al. [56] have shown that the use of Airy beams may extend the field of view in scanned beam light sheet microscopes up to distances $\sim 40$-fold greater than the field of view offered by a Gaussian beam and fourfold that of a Bessel beam.

One key player in the development of fast light sheet microscopy setups has been the development of scientific CMOS (complementary metal oxide semiconductor) cameras capable of imaging at 200 frames per second and higher. By scanning the beam and focusing the sample using a tunable lens, Fahrbach et al. [40] were able to image several full 3D stacks of a beating zebrafish heart per second (Figure 2F,G). Another relevant example of fast scanning in vivo is the approach used in [57] where the authors achieve whole-brain functional imaging in zebrafish (Figure 2C-E) where the neuronal activity within the brain can be resolved with cellular resolution.

In the field of development, light sheet microscopy has had a significant impact, showing in vivo development with cellular resolution of wide range of species. As examples of specimens being imaged with high quality using light sheet microscopy, we should point out invertebrates such as D. melanogaster [48], C. elegans [58], and T. castaneum [59], and vertebrates such as zebrafish $[47,60]$ (for very useful information regarding zebrafish preparation for laser sheet imaging see [61]). Of particular importance is the work by lchikawa et al. where light sheet microscopy was applied to a developing mouse embryo, given the complexity of keeping a mouse embryo under controlled $\mathrm{CO}_{2}$ and temperature conditions [62]. In this work an approach for transferring embryos to the laser sheet microscope without exposing them to the environment is presented.

Overall, light sheet microscopy has several advantages, the most obvious being the speed of acquisition and the low exposure of the specimens, allowing long-term in vivo imaging without damaging the sample. One major drawback of light sheet microscopy, however, is inherent to its geometry: in those cases where the specimen cannot be accessed from the side, generating a sheet of light which coincides with the focal plane of an objective becomes unpractical, at least when we deviate from surface measurements. Such an instance occurs for example when imaging in vivo in an adult mouse through an imaging window. In such cases multiphoton microscopy will yield the best results for depths up to $1-2 \mathrm{~mm}$. 

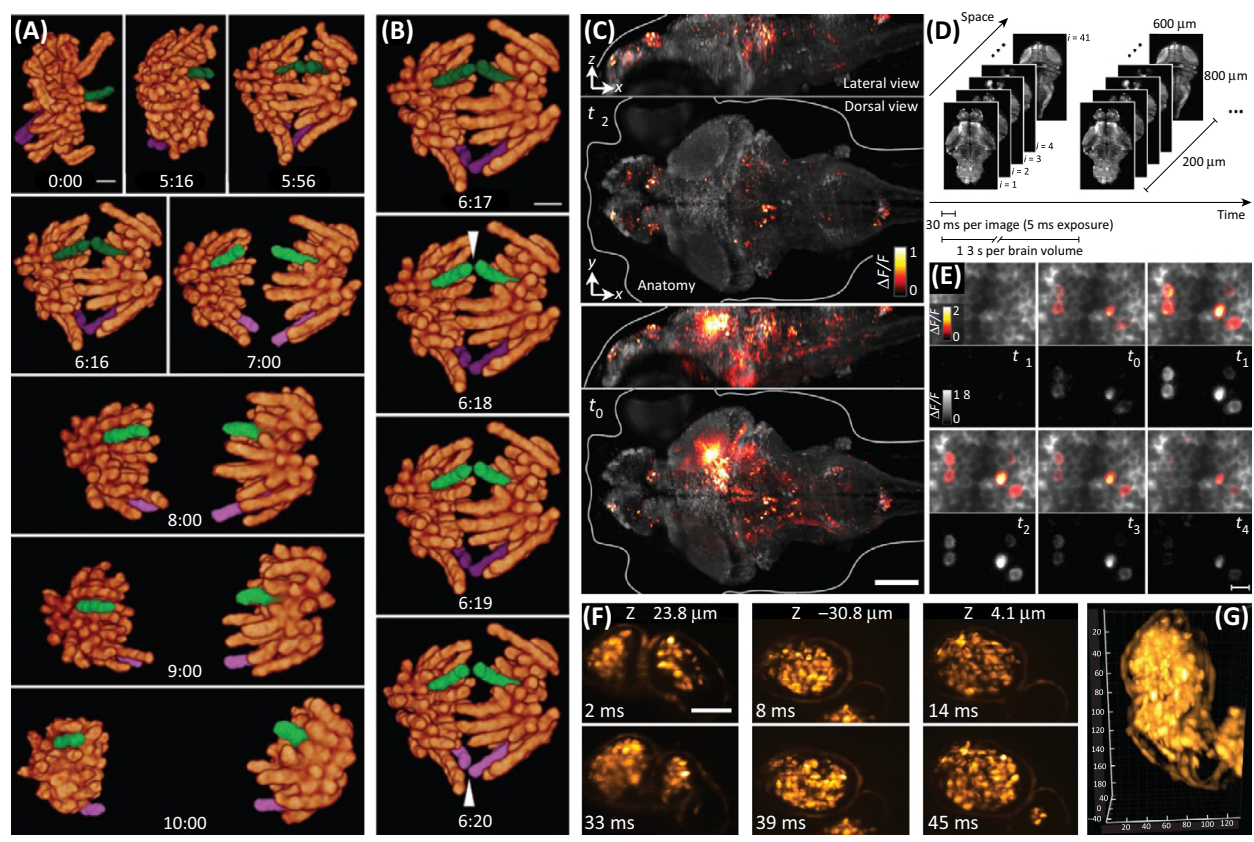

Trends in Biotechnology

Figure 2. (A and B) High speed volumetric imaging of chromosomes in mitosis. (A) Eight image volumes from a series of 200 such volumes depicting mitosis in a LLC PK1 cell transfected with plasmids encoding mEmerald histone H2B and imaged in the Bessel TPE sheet mode. Each volume, composed of 200 image planes, was acquired in $1.0 \mathrm{~s}$. The rest interval between stacks varied from $20 \mathrm{~s}$ in metaphase and telophase to no rest in early anaphase, to expend more of the photon budget at the points of most rapid evolution. Two chromatids (green and purple) are traced through the series. (B) Four consecutive image volumes from the series, during the fast imaging period in anaphase, in which the two chromatids separate (arrowheads). Times indicate minutes:seconds. Scale bars, $5 \mu \mathrm{m}$ (from Planchon et al. [51]). (C E) Whole brain imaging of neuronal activity with cellular resolution. (C) Dorsal and lateral projections of whole brain, neuron level functional activity, reported by the genetically encoded calcium indicator GCaMP5G in an elavl3:GCaMP5G fish via changes in fluorescence intensity (DF/F), superimposed on the reference anatomy. Near simultaneous activation of a large population of neurons occurs at the second frame (t0) and spans areas in the midbrain and hindbrain. (D) High resolution images are recorded in steps of $5 \mu \mathrm{m}$ every $30 \mathrm{~ms}$, with an exposure time of $5 \mathrm{~ms}$ per image. A volume of $800 \times$ $600 \times 200 \mu \mathrm{m}^{3}$, containing the entire brain, is recorded once every $1.3 \mathrm{~s}$. (E) Activity is shown superimposed on the anatomy (top) as well as separate from the anatomy (bottom). Slices represent activity recorded at consecutive intervals of 1.39 s. Scale bars, $100 \mu \mathrm{m}(\mathrm{C}), 10 \mu \mathrm{m}$ (E) (from Ahrens et al. [57]). (F,G) Fast 3D imaging of the beating zebrafish heart. Data for several different planes in a beating zebrafish heart can be acquired almost synchronously through the use of tunable lens. In $(F)$ two images taken in three different planes are shown. The temporal spacing between two frames taken in the same plane is $1 / 30 \mathrm{~s}$. Scale bar, $50 \mu \mathrm{m}$ (from Fahrbach et al. [40]).

\section{Imaging Smaller: Pushing Resolution to the Limit}

Finally, the third aspect of imaging that needs to be addressed is resolution, where we find great efforts directed towards super-resolution techniques, including stimulated emission depletion (STED) [63-66], structured illumination microscopy (SIM) [67,68], photoactivated localization microscopy (PALM) [69,70], and stochastic optical reconstruction microscopy (STORM) [71-74]. These approaches offer sub-diffraction-limited resolution and have opened new ways of exploring the submicron world within the living cell.

In conventional light microscopy the lateral and axial resolution depends on the size of the focal spot (the point spread function, PSF) which, in the absence of scattering, is limited by diffraction. In the lateral direction this size is approximately $\lambda / 2$, where $\lambda$ is the wavelength of the excitation source. In the axial direction the resolution of a confocal microscope worsens due to the limited aperture angle which causes the extension of the focal spot in the optical axis to be about threeto fourfold larger than in the lateral direction [1]. 
In STED microscopy [66,75], in addition to the excitation beam a second doughnut-shaped beam is coupled into the illumination pathway. STED uses saturated stimulated depletion to transfer all excited fluorescent molecules back to the ground state, with the exception of the small volume present at the central focal point (i.e., the 'hole' of the doughnut), its size being nonlinearly related to the depletion intensity. The STED approach can be extended to a more general principle as shown in reversible saturable/switchable optically linear fluorescence transition (RESOLFT) microscopy [76], which can be performed with multiple kinds of switchable fluorescent molecules, slightly reducing the intensity needed for imaging. With STED a resolution of $70 \mathrm{~nm}$ has been reached in living cells [65,77], as can be seen in Figure 3E where a two-color STED microscope is used to image a living HEK293 cell over an interval of more than 4 minutes [65]. Drawbacks of targeted switching techniques such as STED and RESOLFT are that they often require high intensities, resulting in high photo-bleaching and photo-toxic effects. A higher resolution is always accompanied a trade-off in acquisition time, where for a scanning method such as STED the step size has to be smaller than half of the desired resolution.

In PALM [78,79] and STORM [71], individual molecules are switched on sparsely over the whole field of view by stochastic light activation. Even though targeted switching with STED and RESOLFT, and stochastic switching with PALM or STORM, have opened new possibilities in the field of cell-, micro-, and neurobiology through their superior resolution, they also have some drawbacks. For stochastic switching methods the acquisition speed is even more limited. The first restricting factor is that only a small fraction of the fluorophores can be activated simultaneously to ensure that the distance between them is large enough for individual localization. The second restricting factor is that the accuracy depends on the number of collected photons and therefore on the exposure time. However, one of the benefits of PALM and STORM compared to targeted switching methods such as STED and RESOLFT is that lower intensities are needed.

A technique which offers a superior acquisition time at lower intensities and still gives subdiffraction resolution is (3D) SIM (3D-SIM) $[67,68,80,81]$, which improves the diffraction-limited resolution by a factor of about two (note that STED and PALM/STORM offer higher resolution). In SIM the sample is illuminated by a pattern which is rotated and translated, and all this information is combined to generate an image with twice the resolving power than that obtained with homogeneous illumination, with a slight increase in acquisition times (one image per rotation, typically). In Figure 3F, 3D-SIM images of DNA, nuclear lamina, and nuclear pore complex (NPC) epitopes are compared to an image from a confocal laser scanning microscope showing how 3D-SIM offers superior resolution while still allowing in vivo imaging. Another implementation of SIM is shown in Figure 3A-D [82], where the sample is illuminated with a structured (lattice) light sheet, enabling fast imaging at resolutions beyond the diffraction limit, a technique which can also be combined with PALM (Figure 3C,D).

\section{Getting The Best of All Worlds: Combining Microscopy With Optoacoustics}

With the availability of a variety of high-performance optical imaging methods, the selection and investment into an imaging toolbox for biological discovery becomes a daunting task (Figure 4, Key Figure, gives a comparison of the new technologies available and details on their requirements). The biologist of past decades needed, at most, to specify a few parameters such as wavelength range and magnification capacity. Modern scientists, however, require an in-depth understanding of a large range of techniques to optimally decide on the tools of interest. Central to this decision is the research strategy and focus.

With respect to depth, biology has been limited to the observation of superficial events or transparent organisms, this limitation having a direct effect on which research lines may be pursued. However, the optoacoustic methods open up new possibilities for imaging deeper structures or even small animals. As mentioned previously, the depth penetration of all-optical 

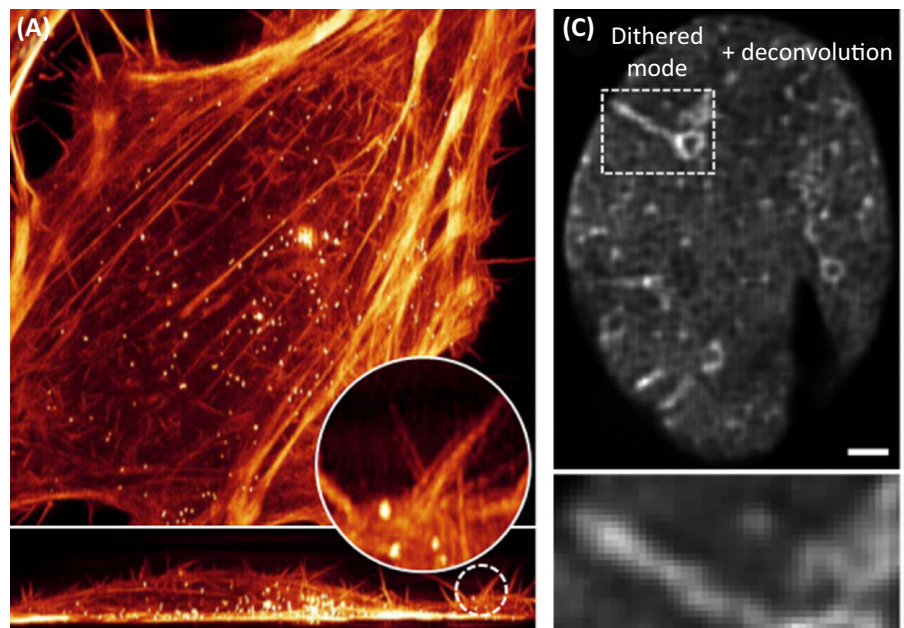

(D) Lattice light sheet PALM
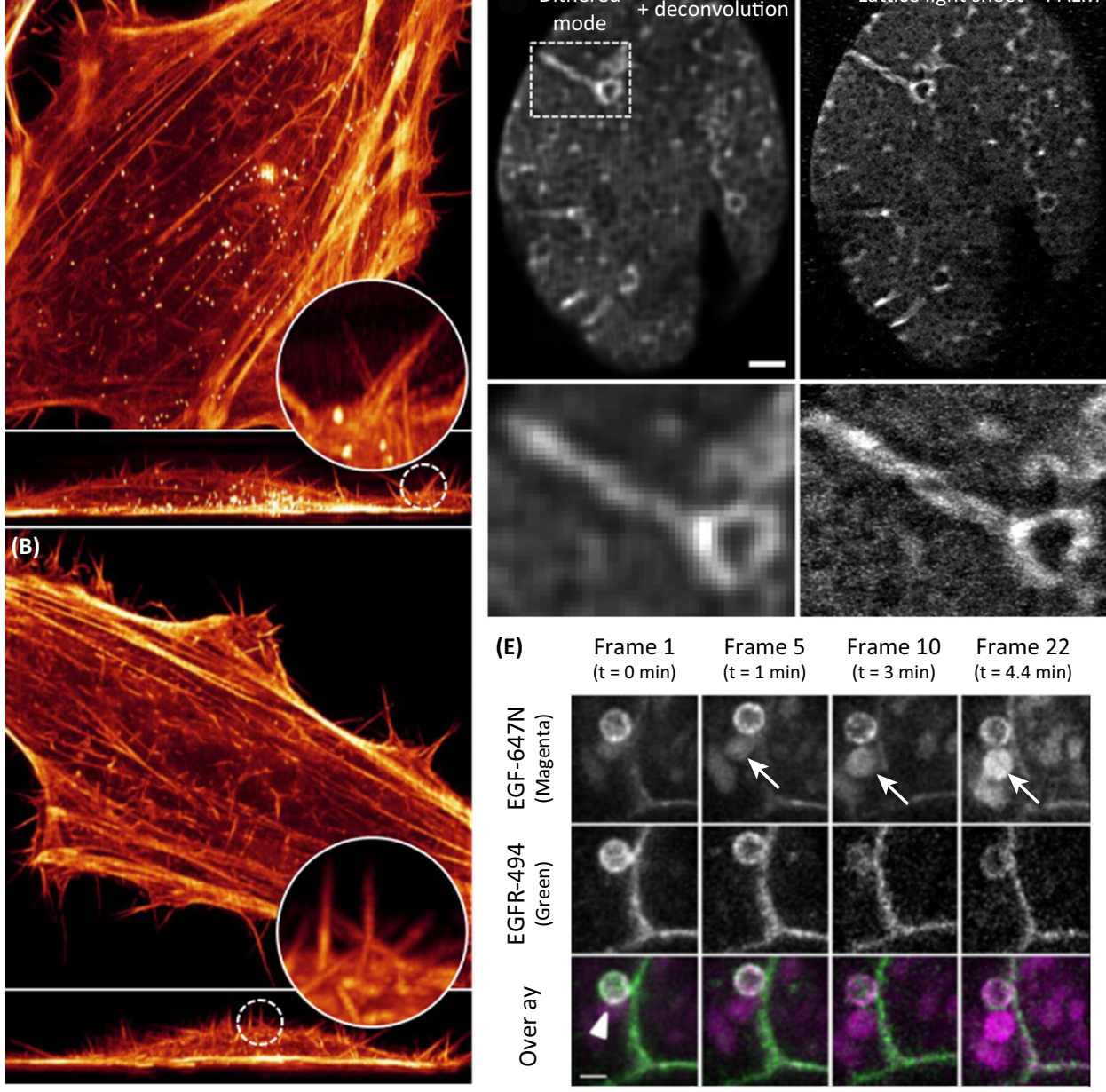

(F)
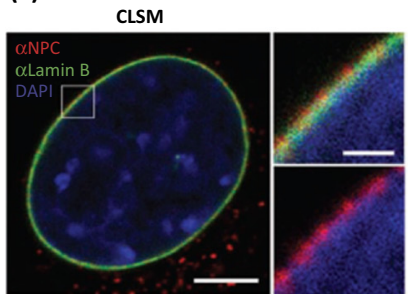

CLSM + deconvolution
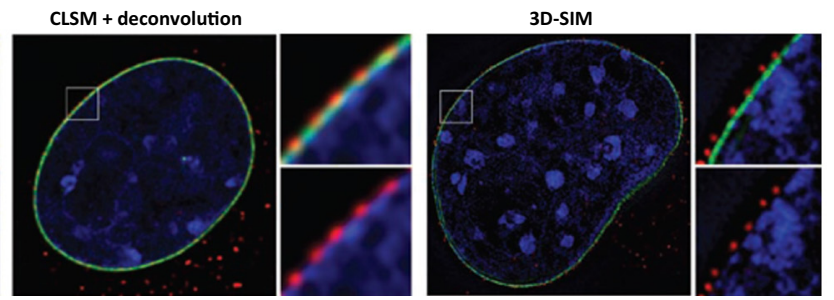

Trends in Biotechnology

Figure 3. (A and B) Experimental comparisons of Bessel beam and lattice light sheet microscopy. (A) 3D renderings in the $x y$ (top) and $x z$ (bottom) directions of the 300th timepoint of a 4D dataset of a living HeLa cell transfected with mEmerald Lifeact, taken in the SIM mode with a five phase hexagonal lattice at $7.5 \mathrm{~s}$ intervals. (B) The 300th timepoint from a third HeLa cell, acquired with a hexagonal lattice in the dithered mode at $1.5 \mathrm{~s}$ intervals. Scale bar, $5 \mu \mathrm{m}$. The low phototoxicity of this mode permits even light sensitive specimens such as $D$. discoideum to be imaged for long periods (from Chen et al. [82]). (C and D) PALM super resolution. (C) Diffraction limited maximum intensity projection (MIP) from a slab $600 \mathrm{~nm}$ in thickness cut through the bottom of the nuclear membrane of a fixed U2OS cell expressing Dendra2 lamin A, taken in the dithered lattice mode. (D) Super resolution MIP from the same slab, taken with 3D PALM, where molecules in successive planes are excited with a dithered lattice light sheet. Scale bars, $2 \mu \mathrm{m}$ in upper views of (C) and (D), $1 \mu \mathrm{m}$ in zoomed boxes below (from Chen et al. [82]). (E) STED super resolution. Live cell two color STED time series of HEK293 cells labeled with EGF CLIPf ATTO647N (magenta) and EGFR SNAPf Chromeo494 (green). Data have been normalized to correct for bleaching. The images shown have been cropped from the original raw data. Scale bar $1 \mu \mathrm{m}$ (from Pellett et al. [65]). (F) Simultaneous imaging of DNA, nuclear lamina, and nuclear pore complex (NPC) epitopes by 3D SIM. C2C12 cells are immunostained with antibodies against lamin B (green) and antibodies that recognize different NPC epitopes (red). DNA is counterstained with DAPI (blue). Central cross sections from Schermelleh et al. [81]. 


\section{Key Figure}

\section{Comparison of Imaging Modalities Used in Developmental Studies}

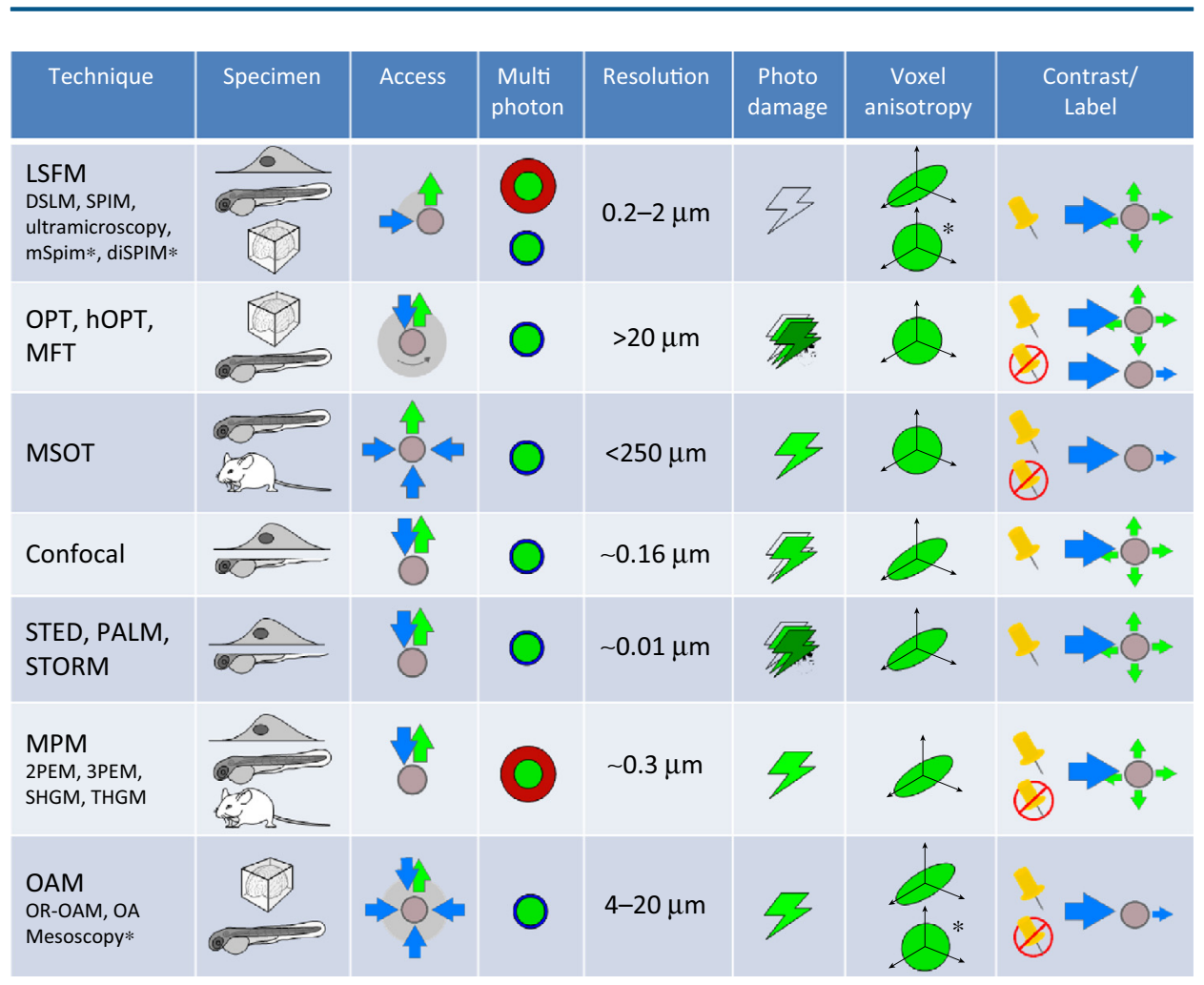

*Isotropy reached through multiple views

(Cleared) whole organs

Trends in Biotechnology

Figure 4. Comparison of novel imaging modalities based on the type of specimen they can image, the illumination/detection geometry, their multiphoton capabilities, resolution, photodamage, voxel anisotropy, and the contrast or label needed to generate an image. Note that in all optical imaging approaches resolution will worsen as scattering increases. Abbreviations: hOPT, helical optical projection tomography; LSM, light sheet microscopy; MFT, mesoscopic fluorescence tomography; MPM multiphoton microscopy; MSOT, multispectral optoacoustic tomography; OAM, optoacoustic microscopy; PALM, photo activated localization microscopy; STED, stimulated emission depletion; STORM, stochastic optical reconstruction microscopy. 
imaging modalities is approximately one transport mean free path ( $\sim 1 \mathrm{~mm}$ in tissues). While multiphoton microscopy and laser sheet microscopy have already extended our ability to image structures to that limit, optoacoustics increases this range even further to the range of several centimeters (i.e., 10-100 mean free paths). One additional advantage that optoacoustic imaging offers is label-free imaging together with the possibility of using contrast agents which will render processes within the organism visible, or the ability to perform functional imaging by distinguishing between the absorption spectrum of endogenous molecules such as oxy- and deoxyhemoglobin.

Sometimes the requirements for an experiment cannot be fulfilled by a single technique. At this point a hybrid system combining two or more imaging modalities may be the solution. Optical techniques with similar geometries can be combined fairly easily and provide additional contrast $[7,9,12]$. However, the combination of two similar optical techniques will usually not improve the imaging depth significantly. One possibility for future systems would be to combine optical and optoacoustic systems so as to benefit from both the high resolution of an optical microscope and the larger imaging depth of an optoacoustic system. It has already been shown that optoacoustic microscopy can be integrated into an confocal or multiphoton microscope and deliver complementary contrast [83-85], but the full potential of combining the two techniques has not been reached yet.

\section{Concluding Remarks and Future Perspectives}

In summary, the main approach to reduce the effect of scattering and photobleaching is to design systems that are capable of separating light that has suffered very little scattering (ballistic light) from highly scattered light. This is achieved by tuning one or several of the parameters of our light sources, such as the spatial coherence through interference or structured illumination, the temporal profile of the pulse (detecting early arriving light, for example), or wavelengths that present larger penetration depths and generate non-linear processes (such as multiphoton excitation, second or third harmonic generation, and Raman scattering). These different forms of illumination, when combined with different detection geometries and methodologies, give rise to specific imaging modalities. In this review we have presented the most recent development in techniques that are more relevant to developmental biology because of their capacity to provide volumetric information on developing organisms. We have seen how these different techniques work and how they are affected by, and account for, scattering, showing how (and why) each of these techniques has a range of applications in which they are extremely useful. We believe that merging microscopy and optoacoustics shows great potential for imaging in developmental biology to explore those regions which are still inaccessible to mainstream imaging modalities, generating high-resolution images ranging in scale from the microscopic to the macroscopic (see Outstanding Questions).

\section{Acknowledgments}

The work on this review article has received funding from the Deutsche Forschungsgemeinschaft (DFG), Germany (Leibniz Prize 2013; NT 3/10 1) and the Federal Ministry of Education and Research (BMBF), Photonic Science Germany, Tech2See 13N12623/ 4. J.R. acknowledges support from the European Commission FP7 CIG grant HIGH THROUGH PUT TOMO, and Spanish MINECO grant MESO IMAGING FIS2013 41802 R.

References

1. Pawley, J. (2010) Handbook of Biological Confocal Microscopy, 5. Min, W. et al. (2011) Coherent nonlinear optical imaging: beyond Springer

2. Ripoll, L.J. (2012) Principles of Diffuse Light Propagation: Light Propagation in Tissues with Applications in Biology and Medicine, World Scientific

3. Hong, G. et al. (2014) Through-skull fluorescence imaging of the brain in a new near-infrared window. Nat. Photonics 8, 723-730

4. Hoover, E. and Squier, J. (2013) Advances in multiphoton microscopy technology. Nat. Photonics 7, 93-101
Outstanding Questions

Full 3D super resolution microscopy in vivo: can full 3D super resolution imag ing approaches be developed that are capable of imaging several layers of live cells while maintaining resolution within tens of nanometers?

Development of NIR Ila fluorophores: can the $13001400 \mathrm{~nm}$ region of the spectrum be exploited for in vivo imag ing in small animals through the devel opment of activatable fluorophores specific for this spectral region?

Fast adaptive optics: will it be possible to use real time adaptive optics and wavefront correction devices to improve resolution within live tissues, enabling fast illumination and detection correction at frequencies in the $\mathrm{MHz}$ range?

Optoacoustic hybrid imaging systems: can we develop of a combined struc tured illumination laser sheet micro scope combined with an optoacoustic imaging system that will be capable of time lapse imaging from the first cellular division to the adult animal? 
9. Mahou, P. et al. (2012) Multicolor two-photon tissue imaging by wavelength mixing. Nat. Methods 9, 815-818

10. Cia, D. et al. (2013) New Tools for the brainbow toolbox. Nat. Methods 10, 540-547

11. Friedl, P. et al. (2007) Biological second and third harmonic generation microscopy. Curr. Protoc. Cell Biol. 4, 4.15

12. Witte, S. et al. (2011) Label-free live brain imaging and targeted patching with third-harmonic generation microscopy. Proc. Natl. Acad. Sci. U.S.A. 108, 5970-5975

13. Olivier, N. et al. (2010) Cell lineage reconstruction of early zebrafish embryos using label-free nonlinear microscopy. Science 329, 967-971

14. Birk, U.J. et al. (2011) Improved reconstructions and generalized filtered back projection for optical projection tomography. Appl. Opt. 50, 392-398

15. Walls, J.R. et al. (2005) Correction of artefacts in optical projection tomography. Phys. Med. Biol. 50, 4645-4665

16. Sharpe, J. et al. (2002) Optical projection tomography as a tool for 3D microscopy and gene expression studies. Sc ence 296, 541-545

17. Bassi, A. et al. (2011) In vivo label-free three-dimensional imaging of zebrafish vasculature with optical projection tomography. J. Biomed. Opt. 16, 100502

18. Fieramonti, L. et al. (2012) Time-gated optical projection tomog raphy allows visualization of adult zebrafish internal structures. PLOS ONE 7, e50744

19. Colas, J. and Sharpe, J. (2009) Live optical projection tomography. Organogenesis 5, 211-216

20. Rieckher, M. et al. (2011) Microscopic optical projection tomography in vivo. PLOS ONE 6, 2-7

21. Arranz, A. et al. (2013) Helical optical projection tomography. Opt Expr. 21, 25912-25925

22. Arranz, A. et al. (2014) In vivo optical tomography of small scattering specimens: time-lapse $3 \mathrm{D}$ imaging of the head eversion process in Drosophila melanogaster. Sci. Rep. 4, 7325

23. Vinegoni, C. et al. (2008) In vivo imaging of Drosophila melanogaster pupae with mesoscopic fluorescence tomography. Nat. Methods 5, 2007-2009

24. McGinty, J. et al. (2008) Fluorescence lifetime optical projection tomography. J. Biophotonics 394, 390-394

25. Fieramonti, L. et al. (2013) Quantitative measurement of blood velocity in zebrafish with optical vector field tomography. J. Biophotonics 8, 51-59

26. Treeby, B.E. et al. (2010) Photoacoustic tomography in absorbing acoustic media using time reversal. Inverse Probl. 26, 115003

27. Rosenthal, A. et al. (2010) Fast semi-analytical model-based acoustic inversion for quantitative optoacoustic tomography. IEEE Trans. Med. Imaging 29, 1275-1285

28. Lao, Y. et al. (2008) Noninvasive photoacoustic imaging of the developing vasculature during early tumor growth. Phys. Med. Biol. 53, 4203-4212

29. Laufer, J. et al. (2007) Quantitative spatially resolved measurement of tissue chromophore concentrations using photoacoustic spectroscopy: application to the measurement of blood oxygenation and haemoglobin concentration. Phys. Med. Biol. 52, 141-168

30. Razansky, D. et al. (2007) Multispectral photoacoustic imaging of fluorochromes in small animals. Opt. Lett. 32, 2891

31. Ntziachristos, V. and Razansky, D. (2010) Molecular imaging by means of multispectral optoacoustic tomography (MSOT). Chem. Rev. 110, 2783-2794

32. Razansky, D. et al. (2011) Volumetric real-time multispectral optoacoustic tomography of biomarkers. Nat. Protoc. 6, 1121-1129

33. Ma, R. et al. (2009) Multispectral optoacoustic tomography (MSOT) scanner for whole-body small animal imaging. Opt. Express 17, 21414-21426

34. Razansky, D. et al. (2009) Multispectral opto-acoustic tomography of deep-seated fluorescent proteins in vivo. Nat. Photonics 3 , 412-417

35. Gateau, J. et al. (2013) Ultra-wideband three-dimensional optoacoustic tomography. Opt. Lett. 38, 4671-4674

36. Omar, A.M. et al. (2014) Ultra-wideband reflection-mode optoacoustic mesoscopy. Opt. Lett. 39, 3911-3914
37. Light-Sheet Microscopy, December 2013. Nat. Collect. 1,

38. Mahou, P. et al. (2014) Multicolor two-photon light-sheet microscopy. Nat. Publ. Gr. 11, 600-601

39. Mickoleit, M. et al. (2014) High-resolution reconstruction of the beating zebrafish heart. Nat. Methods 11, 919-922

40. Fahrbach, F. et al. (2013) Rapid 3D light-sheet microscopy with a tunable lens. Opt. Express 21, 1963-1975

41. Siedentopf, H. and Zsigmondy, R. (1903) Über Sichtbarmachung und Größenbestimmung ultramikroskopischer Teilchen, mit besonderer Anwendung auf Goldrubingläser. Annalen Der Physik 10, 1-39

42. Voie, A.H. et al. (1993) Orthogonal-plane fluorescence optica sectioning: three-dimensional imaging of macroscopic biological specimens. J. Microsc. 170, 229-236

43. Fuchs, E. et al. (2002) Thin laser light sheet microscope for microbial oceanography. Opt. Express 10, 1131-1140

44. Dodt, H. et al. (2007) Ultramicroscopy: three-dimensional visualization of neuronal networks in the whole mouse brain. Nat. Meth ods 4, 331-336

45. Huisken, J. et al. (2004) Optical sectioning deep inside live embryos by selective plane illumination microscopy. Science 305, 1007-1009

46. Weber, M. et al. (2013) Light sheet microscopy. Methods Cell Biol. $123,193-215$

47. Keller, P. et al. (2008) Reconstruction of zebrafish early embryonic development by scanned light sheet microscopy. Science 322 , 1065-1069

48. Keller, P.J. et al. (2010) Fast, high-contrast imaging of anima development with scanned light sheet-based structured-illumination microscopy. Nat. Methods 7, 637-642

49. Gao, L. et al. (2014) 3D live fluorescence imaging of cellular dynamics using Bessel beam plane illumination microscopy. Nat. Protoc. 9, 1083-1101

50. Gao, L. et al. (2012) Noninvasive Imagng beyond the diffraction limt of 3D dynamcs in thickly fluorescent specimens. Cell 151, 1370-1385

51. Planchon, T.A. et al. (2011) Rapid three-dimensional isotropic imaging of living cells using Bessel beam plane illumination. Nat. Methods 8, 417-423

52. Swoger, J. et al. (2007) Multi-view image fusion improves resolution in three-dimensional microscopy. Opt. Express 15, 8029

53. Preibisch, S. et al. (2010) Software for bead-based registration of selective plane illumination microscopy data. Nat. Methods 7 , 418-419

54. Krzic, U. et al. (2012) Multiview light-sheet microscope for rapid in toto imaging. Nat. Methods 9, 730-733

55. Wu, Y. et al. (2013) Spatially isotropic four-dimensional imaging with dual-view plane illumination microscopy. Nat. Biotechnol. 31 , 1032-1038

56. Vettenburg, T. et al. (2014) Light-sheet microscopy using an Airy beam. Nat. Methods 11, 541-544

57. Ahrens, M. and Keller, P. (2013) Whole-brain functional imaging at cellular resolution using light-sheet microscopy. Nat. Methods 10, 413-420

58. Wu, Y. and Ghitani, A. (2011) Inverted selective plane illumination microscopy (ISPIM) enables coupled cell identity lineaging and neurodevelopmental imaging in Caenorhabditis elegans. Proc. Natl. Acad. Sci. U.S.A. 108, 17708-17713

59. Strobl, F. and Stelzer, E.H.K. (2014) Non-invasive long-term fluorescence live imaging of Tribolium castaneum embryos. Develop ment 141, 2331-2338

60. Ahrens, M.B. et al. (2013) Whole-brain functional imaging at cellular resolution using light-sheet microscopy. Nat. Methods 10 413-420

61. Kaufmann, A. et al. (2012) Multilayer mounting enables long-term imaging of zebrafish development in a light sheet microscope. Development 139, 3242-3247

62. Ichikawa, T. et al. (2014) Live imaging and quantitative analysis of gastrulation in mouse embryos using light-sheet microscopy and 3D tracking tools. Nat. Protoc. 9, 575-585

63. Hein, B. et al. (2008) Stimulated emission depletion (STED) nanoscopy of a fluorescent protein-labeled organelle inside a living cell. Proc. Natl. Acad. Sci. U.S.A. 105, 14271-14276 
64. Lukinavicius, G. et al. (2014) Fluorogenic probes for live-cell imaging of the cytoskeleton. Nat. Methods 11, 731-733

65. Pellett, P. et al. (2011) Two-color STED microscopy in living cells. Biomed. Opt. 2, 8186-8193

66. Willig, K.I. et al. (2006) Nanoscale resolution in GFP-based microscopy. Nat. Methods 3, 721-723

67. Heintzmann, R. and Cremer, C. (1999) Laterally modulated excitation microscopy: Improvement of resolution by using a diffraction grating. Proc. SPIE 3568, 185-196

68. Gustafsson, M.G.L. et al. (1998) Three-dimensional fluorescence microscopy beyond the classical resolution limit. INT CON Electron. Microsc. Mater. Sci. 3, 71

69. Shroff, H. et al. (2008) Live-cell photoactivated localization microscopy of nanoscale adhesion dynamics. Nat. Methods 5, 417-423

70. Achurra, P. et al. (2014) Photoactivated localization microscopy for cellular imaging. In Super-Resolution Microscopy Techniques in the Neurosciences (Fornasiero, E. and Rizzoli, S., eds), pp. 87111, Springer

71. Rust, M.J. et al. (2006) Sub-diffraction-limit imaging by stochastic optical reconstruction microscopy (STORM). Nat. Methods 3, 793-795

72. Xu, K. et al. (2012) Dual-objective STORM reveals three-dimensional filament organization in the actin cytoskeleton. Nat. Methods 9, 185-188

73. Jones, S. et al. (2011) Fast, three-dimensional super-resolution imaging of live cells. Nat. Methods 8, 499-505

74. Heilemann, M. et al. (2008) Subdiffraction-resolution fluorescence imaging with conventional fluorescent probes. Angew. Chem. Int. Ed. Engl. 47, 6172-6176

75. Hell, S.W. and Wichmann, J. (1994) Breaking the diffraction resoIution limit by stimulated emission: stimulated-emission-depletion fluorescence microscopy. Opt. Lett. 19, 780-782

76. Schwentker, M. and Bock, H. (2007) Wide-field subdiffraction RESOLFT microscopy using fluorescent protein photoswitching. Microsc. Res. 280, 269-280

77. Berning, S. et al. (2012) Nanoscopy in a living mouse brain. Science 335, 55
78. Betzig, E. et al. (2006) Imaging intracellular fluorescent proteins at nanometer resolution. Science 313, 1642-1645

79. Hess, S.T. et al. (2006) Ultra-high resolution imaging by fluorescence photoactivation localization microscopy. Biophys. J. 91 , 4258-4272

80. Gustafsson, M.G. (2000) Surpassing the lateral resolution limit by a factor of two using structured illumination microscopy. J. Microsc $198,82-87$

81. Schermelleh, L. et al. (2008) Subdiffraction multicolor imaging of the nuclear periphery with $3 \mathrm{D}$ structured illumination microscopy. Science 320, 1332-1336

82. Chen, B-C. et al. (2014) Lattice light-sheet microscopy: Imaging molecules to embryos at high spatiotemporal resolution. Science 346, 1257998

83. Tserevelakis, G.J. et al. (2014) Hybrid multiphoton and optoacoustic microscope. Opt. Lett. 39, 1819-1822

84. Wang, Y. et al. (2011) In vivo integrated photoacoustic and confocal microscopy of hemoglobin oxygen saturation and oxygen partial pressure. Opt. Lett. 36, 1029-1031

85. Langer, G. et al. (2013) Two-photon absorption-induced photoacoustic imaging of Rhodamine B dyed polyethylene spheres using a femtosecond laser. Opt. Express 21, 22410-22422

86. Zhou, E.H. et al. (2014) Focusing on moving targets through scattering samples. Optica 1, 227-232

87. Ruan, H. et al. (2014) Iterative time-reversed ultrasonically encoded light focusing in backscattering mode. Nat. Sci. Rep. $4,1-7$

88. Dalgarno, H.I.C. et al. (2012) Wavefront corrected light sheet microscopy in turbid media. Appl. Phys. Lett. 100, 191108

89. Vellekoop, I.M. and Mosk, A.P. (2007) Focusing coherent light through opaque strongly scattering media. Opt. Lett. 32, 2309-2311

90. Chaigne, T. et al. (2014) Light focusing and two-dimensional imaging through scattering media using the photoacoustic transmission matrix with an ultrasound array. Opt. Lett. 39, 2664-2667

91. Wang, K. et al. (2014) Rapid adaptive optical recovery of optima resolution over large volumes. Nat. Methods 11, 625-628 